\title{
DISTRIBUCIÓN ESPACIAL DE LA SALINIDAD EN SUELOS DEL ÁREA DE INFLUENCIA DE LA DESEMBOCADURA DEL RíO SINÚ (CÓRDOBA, COLOMBIA)
}

\section{SPATIAL DISTRIBUTION OF THE SOIL SALINITY IN THE AREA OF INFLUENCE OF THE SINÚ RIVER MOUTH (CORDOBA-COLOMBIA)}

\author{
Humberto Narváez M. ${ }^{1}$, Enrique Combatt C. ${ }^{2}$, Iván Bustamante Barrera ${ }^{3}$
}

\begin{abstract}
${ }^{1}$ Ingeniero Agrónomo. M.Sc. Topografía. Profesor Ocasional Universidad de Córdoba, e-mail: hnarvaez34@hotmail.com; ${ }^{2}$ Ingeniero Agrónomo, Ph.D. Manejo de suelos y nutrición de cultivos. Profesor titular Universidad de Córdoba, e-mail: ecombatt@fca.edu.co, dirección para correspondencia; ${ }^{3}$ Ingeniero Agrónomo. M.Sc. en suelos. Profesor titular Universidad de Córdoba, e-mail: ivandabusta@gmail.com, Carrera 6a \# 76-103. Telefax 57-4-7860255. Montería, Córdoba, Colombia.
\end{abstract}

Rev. U.D.C.A Act. \& Div. Cient. 17(2): 433-443, Julio-Diciembre, 2014

\section{RESUMEN}

El área de influencia agropecuaria del delta del río Sinú (Córdoba, Colombia) tiene alto riesgo de salinización, condición química que disminuye la actividad productiva agrícola en esta región. El objetivo del trabajo fue estimar la clase, la magnitud y la distribución espacial de suelos salinos en los últimos $22 \mathrm{~km}$ de la desembocadura del río, en periodos secos y lluviosos. Para ello, se subdividió la zona de estudio en cuatro localidades, donde se colectaron muestras de suelos, hasta $50 \mathrm{~cm}$ de profundidad, en 39 puntos, durante los años 2002-2007. En las muestras colectadas, se evaluó la conductividad eléctrica (CE), $\mathrm{Ca}, \mathrm{Mg}, \mathrm{Na}, \mathrm{K}, \mathrm{HCO}_{3}$ y $\mathrm{SO}_{4}$. La información fue procesada en el programa ArcGis v. 9.3, para interpolar la Distancia Inversa Ponderada y estimar el área y la distribución espacial de los suelos salinos. Además, los datos de CE y de los iones en periodos secos y lluviosos, para las diferentes profundidades, fueron sometidos a análisis de correlación simple (Pearson) y pruebas de separación de medias Tukey (5\%). Los resultados indicaron que en el periodo seco, se acentúan los problemas de salinidad y, la distribución espacial de suelos salinos, se concentra, principalmente, en la localidad 3 y en los primeros $20 \mathrm{~cm}$ de profundidad. Los iones $\mathrm{Cl}$, Na y $\mathrm{Mg}$ presentaron correlaciones positivas y altamente significativas, con $\mathrm{r}$ de $0,68 * *$ y $0,93 * *$ para las diferentes profundidades en el periodo seco; en el periodo lluvioso, la concentración de $\mathrm{Na}$ y $\mathrm{Mg}$ disminuyó, en tanto que, la concentración de los iones $\mathrm{Cl}$, $\mathrm{Ca}$ y $\mathrm{SO}_{4}$, aumentó.

\section{SUMMARY}

The area of influence of the Sinu River Delta (Cordoba, Colombia) has a high risk of salinization, chemical condition that decreases agricultural productive activity in this region. The aim of the study was to estimate the class, magnitude and spatial distribution of saline soils in the last $22 \mathrm{~km}$ of the river mouth in dry and rainy periods. For this purpose, the study area was subdivided in four locations, where soil samples were collected up to $50 \mathrm{~cm}$ deep at 39 points over the years 2002-2007.In the samples collected, the electrical conductivity (EC), $\mathrm{Ca}, \mathrm{Mg}, \mathrm{Na}, \mathrm{K}, \mathrm{HCO}_{3}$ and $\mathrm{SO}_{4}$ was evaluated. The information was processed in the program ArcGis v. 9.3, to interpolate the Distance Inverse average and estimate the area and spatial distribution of saline soils. Also, data of CE and ions in dry and rainy periods for different depths, were subjected to simple correlation analysis (Pearson) and mean separation tests Tukey (5\%). The results indicated that in the dry period salinity problems are accentuated and the spatial distribution of saline soils is mainly concentrated at site 3 and in the first $20 \mathrm{~cm}$ depth. The $\mathrm{Cl}, \mathrm{Na}$ and $\mathrm{Mg}$ ions had positive and highly significant correlations with r $0.68 * *$ and $0.93 * *$ for different depths in the dry season; in the rainy season the concentration of $\mathrm{Na}$ and $\mathrm{Mg}$ decreased, in both that the concentration of $\mathrm{Cl}, \mathrm{Ca}$ and $\mathrm{SO}_{4}$ ions increased.

Key words: Electrical conductivity, salinity, sodium, delta.

Palabras clave: Conductividad eléctrica, sales, sodio, delta, océano. 


\section{INTRODUCCIÓN}

La salinidad es un proceso complejo de degradación química, que influye sobre cambios significativos, en las propiedades físicas de los suelos. Presenta una distribución heterogénea, variable en el tiempo y espacio, a diferentes escalas (GirónRíos et al. 2009). A nivel mundial, se indica que existen más de 800 millones de hectáreas de suelos afectados por altas concentración de diferentes tipos de sales, por lo que se cataloga como uno de los problemas más importantes de la agricultura. Debido a que la salinidad tienen efectos perjudiciales sobre la germinación de las semillas y el crecimiento de las plantas que, eventualmente, atrofian el crecimiento de las plantas, causándole la muerte (FAO 2008; Ramoliya et al. 2006). Según la FAO (2005), en años recientes, se estimó que 400 millones de hectáreas son afectadas por procesos de salinización y, de 230 millones de hectáreas tecnificadas con riego, el $20 \%$ presentan algún grado de salinización y el $10 \%$, tienen riesgo de salinización, por la formación de diferentes tipos de sales. Amezketa (2006) expone que cada año se salinizan unos 0,5 millones de hectáreas de regadío.

En Colombia, dentro de los procesos de degradación de suelos, la salinización se ha definido como uno de los más importantes, alcanzando cerca del 7,7\% de la superficie del país, equivalente a un área aproximada de $87300 \mathrm{~km}^{2}$ (Otero et al. 2002). Las áreas con mayor cantidad de suelos salinos, se manifiesta en zonas costeras y llanuras de la región Caribe, en los valles interandinos y en las zonas costeras de la región del Pacífico. En el departamento de Córdoba, aproximaciones realizadas por el IDEAM (2002), indican que se presenta más de $7700 \mathrm{~km}^{2}$ con problemas de salinización, que afectan los rendimientos de los cultivos.

En estas condiciones edafológicas, los procesos de salinización o de acumulación de sales en los suelos son causados por procesos naturales o antropogénicos que, según Porta (2007), son denominados primarios o secundarios. Rengasamy (2006) indica que la salinización es un proceso complejo, que implica el movimiento de sales y el agua en los suelos durante los ciclos estacionales y las interacciones con las aguas subterráneas. Además, el proceso de salinización depende del régimen de lluvia, meteorización de minerales y sales almacenadas en aguas superficiales y aguas subterráneas, que pueden proporcionar fuentes adicionales de diferentes sales en regiones costeras, aledañas a los océanos.

La alta concentración de sales en estas áreas ocasiona, en general, disminución del crecimiento de los cultivos, debido al estrés hídrico que las sales de la solución del suelo provocan a las plantas, establecidas en estas condiciones edafológicas. Jenks \& Hasegawa (2005) y Rycroft et al. (2002) indican que las altas concentraciones de sodio en los suelos no sólo perjudican las plantas directamente, sino también degradan la estructura del suelo, disminuyendo la porosidad y la permeabilidad del agua. Asimismo, traen como consecuencia, un deterioro acelerado en la calidad de los suelos, por dispersión de las arcillas o desintegración de agregados, que causan encostramiento y endurecimiento de la superficie del suelo. Estos fenómenos repercute en la infiltración del agua y de la germinación, lo que trae, como consecuencia, una disminución de la productividad de éstos, del rendimiento y de la calidad de las cosechas (Bayuelo-Jiménez et al. 2002).

La mayoría de los productores agrícolas ubicados en estas zonas del departamento de Córdoba, Colombia, consideran las áreas cultivables como uniforme, pero las propiedades del suelo son variables en el espacio y en el tiempo. Debido a la alta heterogeneidad, se necesita conocer la distribución espacial de una o varias características, lo cual, se puede realizar con muestreo de campo y procedimientos de interpolación geográfica, para generar imágenes o mapas temáticos. De acuerdo con Juan et al. (2011), algunos atributos físico-químicos del suelo no pueden ser medidos intensivamente de forma rápida, sencilla o a bajo costo, por lo que recomienda el uso de herramienta matemáticas para hacer frente a esta situación y poder realizar estimación y predicción espacial de variables de interés agrícolas o ambientales, para contribuir con la agricultura de precisión (Allaire et al. 2012; Johann et al. 2004).

En este sentido, el uso de métodos geofísicos para el conocimiento espacial de la salinidad en áreas cultivables es cada vez es más necesario para determinar los patrones de variabilidad espacial de las propiedades del suelo y realizar una evaluación más precisa que permita vislumbrar la magnitud del fenómeno, dadas las implicaciones socioeconómicas y productivas que este representa al disminuir la producción agrícola en estas condiciones edafológicas. El objetivo de la investigación fue estimar la clase, la magnitud y la distribución espacial de suelos en proceso de salinización en el área de influencia de la desembocadura del río Sinú en periodos secos y lluviosos.

\section{MATERIALES Y MÉTODOS}

La zona de estudio corresponde a $82 \mathrm{~km}^{2}$ del área de influencia de la desembocadura del río Sinú, concentrada en los últimos $22 \mathrm{~km}$ de su recorrido y cuyos puntos extremos tienen coordenadas geográficas $9^{\circ} 27^{\prime} 22.3^{\prime \prime}$ de latitud N; 7556'34.5” de longitud W y 9¹9'34.6” de latitud N; $75^{\circ} 51^{\prime} 4.5^{\prime \prime}$ de longitud W.

Esta zona limita al norte con el océano Atlántico, al sur con los corregimientos La Doctrina y Las Cañas, al este con las Ciénagas de Ostional y al oeste por el complejo de lomeríos de José Manuel y Calle Ralita, en comprensión territorial de 
los municipios de San Bernardo del Viento y Lorica, del departamento de Córdoba, Colombia. Las características químicas de los suelos están asociadas a contenido de materia orgánica, que oscila entre 1 y 2,5\% y pH, de 6 y 8,4; además, las marea viva se presenta con pico de variaciones de hasta de 0,60m. De acuerdo a la clasificación de Holdridge (1978), esta área corresponde a la zona de vida bosque seco tropical (bs-T), con regímenes de precipitaciones promedia de $1425 \mathrm{~mm}$ año ${ }^{-1}$, temperatura media de $27,6^{\circ} \mathrm{C}$ y humedad relativa del 84\% (Palencia et al. 2006).

Para el estudio, se realizaron 10 muestreos a diferentes profundidades, en suelos dedicados a actividades agropecuarias, como arroz de riego, palma africana, maíz y cultivos de pan coger, como yuca y hortalizas. Los muestreos correspondieron a la colecta de datos en abril de 2002; enero de 2003; abril y noviembre de 2005; mayo y noviembre de 2006; abril y noviembre de 2007 y abril y noviembre del 2008. La colecta de muestras de suelos, se efectuó en 39 puntos seleccionados, próximos a pozos de observación, distribuidos en 25 pozos en vértices principales, con espaciamiento de $2 \mathrm{~km}$ entre sí y 14 en vértices auxiliares, con espaciamiento de $1 \mathrm{~km}$, respecto a los principales y 5 muestras por pozo de observación, a profundidades de 0-10, 10-20, 20-30, 30-40 y $40-50 \mathrm{~cm}$.

Para la zona de estudio, se definieron los periodos correspondientes a los periodos seco y lluvioso del año, para lo cual, se analizó el registro histórico de precipitación de 16 años (1990 a 2005), de la Estación Meteorológica del municipio de San Bernardo del Viento. Como periodo lluvioso, se consideraron los meses que presentaron más del $10 \%$ de la precipitación pluviométrica media anual, que abarca el periodo de mayo a noviembre (más del $92 \%$ de la lluvia total) y el periodo seco, de diciembre a abril.

Las muestras de suelos fueron procesadas por el Laboratorio de Suelos y Aguas de la Universidad de Córdoba y, en ellas, se determinaron los siguientes parámetros: Conductividad eléctrica en $\mathrm{dS} \mathrm{m}^{-1}$ (CE), en un equipo Metrohm, modelo 712 , por método conductimétrico, cationes $\mathrm{Ca}^{2+}, \mathrm{Mg}^{2+}$ en $\mathrm{cmol}^{+} \mathrm{L}^{-1}$, cuantificados por espectrofotometría de absorción atómica $\mathrm{Na}^{+}$y K${ }^{+}$en cmol. $\mathrm{L}^{-1}$, por espectrofotometría de emisión atómica, en un equipo Pelkin Elmer 3110, aniones $\mathrm{Cl}^{-}$en $\mathrm{cmol}^{+} \mathrm{L}^{-1}$, por titulación volumétrica con nitrato de plata $0,0141 \mathrm{~N}, \mathrm{HCO}_{3}^{-}$y $\mathrm{CO}_{3}^{2-}$ en $\mathrm{cmol}^{+} \mathrm{L}^{-1}$, por titulación volumétrica con $\mathrm{H}_{2} \mathrm{SO}_{4}^{-2} 0,02 \mathrm{~N}^{-2} \mathrm{SO}_{4}^{-2}$ en $\mathrm{cmol}^{+} \mathrm{L}^{-1}$, por el método turbidimétrico.

Se generaron mapas de distribución espacial de áreas, de acuerdo a la clasificación de suelos salinos, propuesta por el US Salinity Laboratory (Richards, 1954) y clasificadas como: i) no salinos, con menos de $2 \mathrm{dS} \mathrm{m}^{-1}$; ii) ligeramente salinos, entre 2 - $4 \mathrm{dS} \mathrm{m}^{-1}$; iii) medianamente salinos, entre 4 - $8 \mathrm{dS}$ $\mathrm{m}^{-1}$; iv) fuertemente salinos, $\left.8-16 \mathrm{dS}^{-1} \mathrm{y}, \mathrm{v}\right)$ extremadamente salinos, mayor de $16 \mathrm{dS} \mathrm{m}^{-1}$. Para ello, la información obtenida fue procesada en el programa ArcGis v. 9.3 y aplicándose el interpolador Distancia Inversa Ponderada (IDW, sigla en inglés), el cual, es un método estadístico que presupone que la variable representada disminuye su influencia en cuanto esté más alejada desde su ubicación de muestra. De igual forma, la aplicación permitió estimar el área correspondiente a cada clase de suelos por salinidad a cada profundidad.

Complementariamente, los datos de CE y de los iones en períodos secos y lluviosos para las diferentes profundidades, fueron sometidos a análisis de correlación simple (Pearson) y pruebas de separación de medias Tukey (5\%), para los iones determinados en las diferentes profundidades, en las cuatro localidades, utilizando el programa estadístico SAS (Statistical Analysis System) v. 9.2.

\section{RESULTADOS Y DISCUSIÓN}

Para los periodos seco y lluvioso fueron observadas variaciones en la distribución y porcentaje de salinización, con la época y profundidad evaluada (Tabla 1). De modo general, para el periodo seco del año, el área de suelos no salinos, oscila entre 28,2 y $54,2 \%$, seguido de las áreas que presentan ligera salinidad, con 23 y $26,4 \%$ y moderada salinidad, con rangos entre 25,6 y $18,1 \%$, sin presentarse una tendencia generalizada de incremento o disminución de la salinidad, cuando se analizan los contenidos de sales a diferentes profundidades. Paralelamente y, de acuerdo con la clasificación de suelos salinos propuesta por el US Salinity Laboratory (Richards, 1954), en esta zona se presentan suelos fuerte y extremadamente salinos, con 17,1 y 6,2\% del área que exhibe estas condiciones. Estas condiciones de salinidad, se presentan a las profundidades de $0-10 \mathrm{~cm}$ y $10-20 \mathrm{~cm}$.

Para el periodo lluvioso, las áreas de suelos no salinos, se incrementan en comparación al periodo seco, con áreas entre el 54,6 y 63\%, mientras que suelos ligeramente (LS), medianamente (MS), fuertemente (FS) y extremadamente (ES) salinos mostraron igual orden, en relación a la magnitud de sus áreas, observando reducción de las áreas con suelos fuerte y extremadamente salinos, al ser comparado con el periodo seco.

Las diferencias entre los periodos pueden obedecer a que en la época seca se presentan las mareas más altas (vivas) y la intrusión de aguas salobres al continente es mayor, pues se reducen los flujos hídricos fluviales en el río Sinú. En estas condiciones, se exponen los máximos valores de salinidad, debido a la intensa evapotranspiración potencial, en este periodo, con una deficiencia de agua de $515,8 \mathrm{~mm}$, que se hace notoria en febrero; además, solamente se registra una 
Tabla 1. Distribución y clasificación de suelos salinos, entre 2002 y 2007, para diferentes profundidades del área de influencia, de la desembocadura del río Sinú.

\begin{tabular}{|c|c|c|c|c|c|}
\hline \multirow{5}{*}{ Priodo Seco: Diciembre - Abril } \\
\hline \multirow{5}{*}{$\operatorname{Pr}(\mathbf{c m})$} & \multicolumn{5}{|c|}{ Salinos } \\
\cline { 2 - 6 } & NS & LS & MS & FS & ES \\
\cline { 2 - 6 } & $\mathbf{5}$ & 23,0 & 25,6 & 17,1 & 6,2 \\
\hline $\mathbf{0 - 1 0}$ & 49,4 & 20,8 & 17,1 & 10,1 & 2,7 \\
\hline $\mathbf{1 0 - 2 0}$ & 52,7 & 24,4 & 15,8 & 5,1 & 2,0 \\
\hline $\mathbf{2 0 - 3 0}$ & 54,2 & 25,2 & 16,1 & 2,0 & 2,5 \\
\hline $\mathbf{3 0 - 4 0}$ & 49,7 & 26,4 & 18,1 & 5,0 & 0,7 \\
\hline $\mathbf{4 0 - 5 0}$ & Periodo Lluvioso: Mayo - Noviembre & \\
\hline & 54,6 & 25,3 & 12,6 & 5,4 & 2,0 \\
\hline $\mathbf{0 - 1 0}$ & 55,5 & 21,5 & 14,6 & 6,2 & 2,2 \\
\hline $\mathbf{1 0 - 2 0}$ & 63,0 & 17,8 & 13,6 & 3,5 & 2,1 \\
\hline $\mathbf{2 0 - 3 0}$ & 60,4 & 19,8 & 13,4 & 4,2 & 2,2 \\
\hline $\mathbf{3 0 - 4 0}$ & 58,6 & 18,3 & 14,6 & 6,3 & 2,3 \\
\hline $\mathbf{4 0 - 5 0}$ & & & & \\
\hline
\end{tabular}

precipitación de 5,2mm (Rangel \& Arellano, 2010) y Ridd \& Stieglitz (2002) determinaron que los rápidos aumentos de salinidad en estuarios en las partes áridas de Australia fueron, en gran medida, el resultado de la destilación del agua por evaporación. En época lluviosa, se presentan los valores mínimos, debido al mayor aporte fluvial dado por las precipitaciones y el aumento en el caudal del río Sinú (CIOH, 1994; Sánchez et al. 1997). Por su parte, CIAF (1985) señala que la geología y la geomorfología de la zona es considerada como estuarina, lo que le imprime, a la misma, una considerable vocación salina.

En estas condiciones, con régimen de temperaturas catalogadas como isohipertérmica y caracterizada por temperaturas mayores a $24^{\circ} \mathrm{C}$ durante todo el año, con texturas francas, franco limosas, arenosas y conductividad hidráulica, comprendidas entre 0,1 y 0,5m/día e infiltración básica, entre 0,6 a $1 \mathrm{~cm} / \mathrm{h}$, se registra una reducida tasa de infiltración de agua y el aumento en algún grado la salinidad. Queiroz et al. (2010) afirman que los suelos salinos tienen gran variabilidad espacial y temporal natural, en virtud de las influencias de las prácticas de manejo utilizadas, profundidad del nivel freático, permeabilidad del suelo, tasa de evapotranspiración, precipitación, salinidad del agua subterránea y otros factores geo-hidrológicos.

En cuanto a la distribución espacial, suelos no salinos y ligeramente salinos, se muestran, principalmente, en las localidades 1 (L1) y 2 (L2), que corresponden a las áreas más retiradas de la costa, mientras que las áreas de suelos fuerte y extremadamente salinos, se concentran, esencialmente, en la localidad 3 (L3) y puntos aislados de las localidades 2 (L2) y 4 (L4) (Figura 1).

$\mathrm{Al}$ respecto, en regiones próximas a las costas marinas, la salinización de suelos, se puede deber a la intrusión del agua de mar, la que por evaporación repetida depositará sales en cantidades excesivas; además, en diferentes condiciones edáficas, la salinidad se puede producir por ascenso capilar de aguas freáticas, ricas en sales solubles y, su acumulación, se origina por evaporación y meteorización de minerales.

Según Perfetti \&Terrel (1989), cuando el agua se evapora de los cuerpos de agua existentes, la concentración de sales se incrementa, debido a que la precipitación pluviométrica del agua de lluvia que entra a la corriente contiene trazas de sales y después de la evaporación del agua acumulada, quedan nueva cantidades de sales en el suelo. Asimismo, estas sales pueden ser transportadas, nuevamente, por el flujo superficial del agua de escorrentía, durante las lluvias irregulares a los ríos o al mar.

Para el periodo seco, la distribución espacial de las áreas, de acuerdo a la clasificación de suelos salinos, puede ser explicada, en gran parte, por los contenidos de iones, potencialmente formadores de sales, determinados para las diferentes localidades. En este estudio, los suelos fuerte y extremadamente salinos, se concentran en la localidad 3 (L3), donde, en promedio, se registran altas concentraciones de $\mathrm{Na}, \mathrm{Cl}$ y $\mathrm{Mg}$ y niveles medios, para los iones $\mathrm{Ca}, \mathrm{HCO}_{3}$ y $\mathrm{SO}_{4}$. Gonçal- 

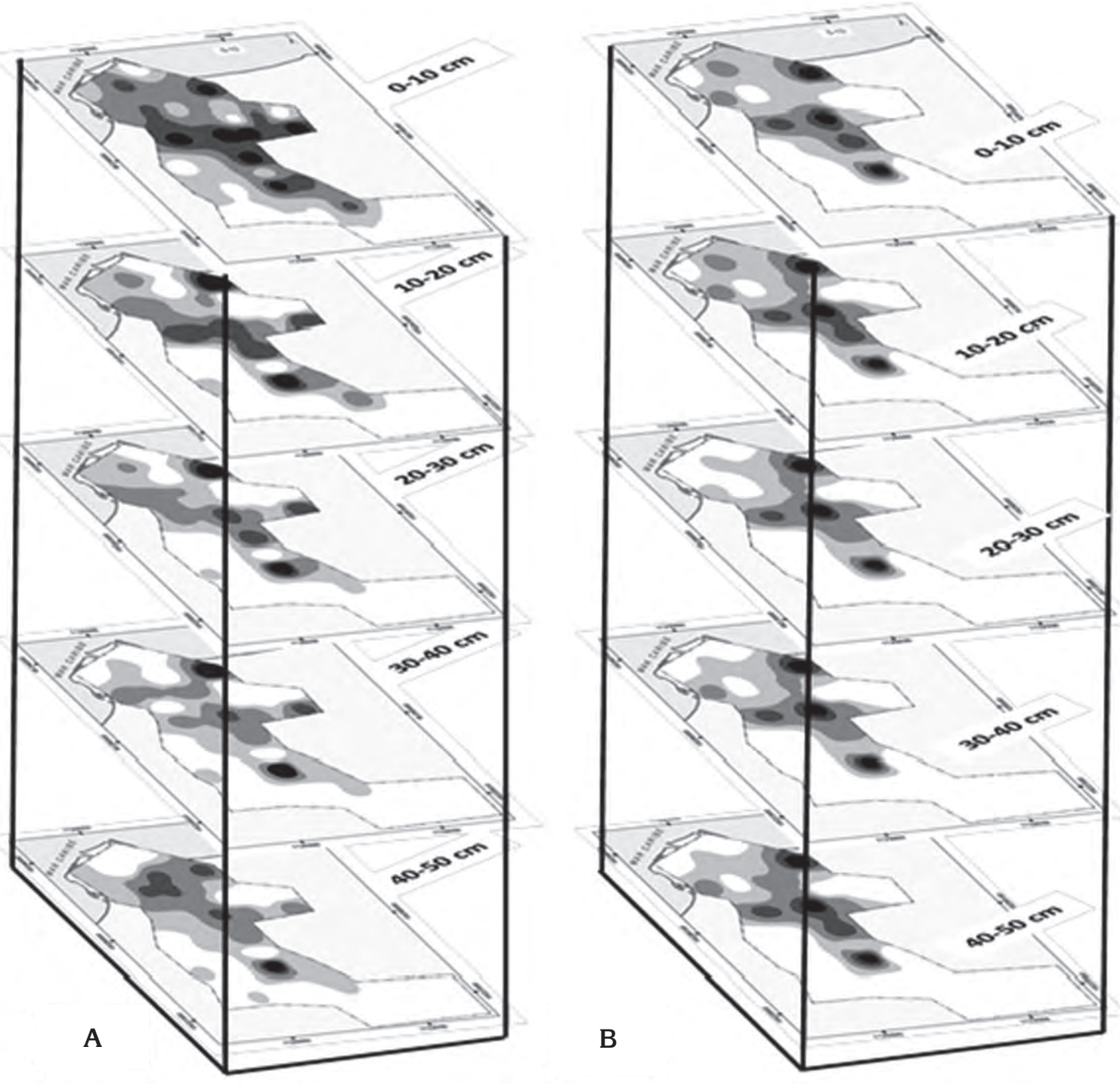

NO SALINO
LIGERAMENTE SALINO
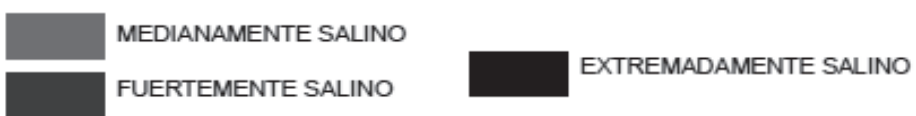

Figura 1. Distribución espacial, de acuerdo a la clasificación de suelos salinos del área de influencia, de la desembocadura del río Sinú. A) Periodo seco y B) Periodo lluvioso.

ves et al. (2011) indican que las sales en exceso perjudican el comportamiento germinativo, vegetativo y productivo de los cultivos, ocasionados por la acción directa de las sales sobre el potencial osmótico del agua y el efecto de los iones, potencialmente tóxicos, como el $\mathrm{Na}, \mathrm{SO}_{4}, \mathrm{Cl}$.

Entre las profundidades evaluadas para el periodo seco, las mayores concentraciones de iones que forman sales, a excepción de la localidad 1 , se registran en la profundidad de
0-10 cm, de tal forma, que los iones Na que se encontraron en esos niveles, que oscilan entre 31,9 y $46,9 \mathrm{cmol} \mathrm{L}^{-1}$ y Cl, se encuentran en niveles excesivos, con 69,7 y $86,8 \mathrm{cmol} \mathrm{L}^{-1}$, niveles altos de $\mathrm{Mg}, \mathrm{Ca}, \mathrm{SO}_{4}$, con concentraciones máxima de 18,2; 20,1 y 29,7 y niveles medios en $\mathrm{HCO}_{3}$, con 5,5 a la misma profundidad (Tabla 2). Esta particularidad de mayor acumulación de sales, se puede relacionar con la característica de solubilidad en agua, que poseen los ion $\mathrm{Na} \mathrm{y} \mathrm{Cl}$. 
Tabla 2. Concentración de iones estudiados en el extracto de saturación, para diferentes localidades y profundidades de suelos, de la desembocadura del río Sinú, en periodo seco 2002 al 2007.

\begin{tabular}{|c|c|c|c|c|c|c|c|}
\hline \multicolumn{8}{|c|}{ Localidad 1: Puntos de muestreo 1 al 13} \\
\hline profundidad & $\mathrm{Ca}$ & $\mathrm{Mg}$ & $\mathrm{Na}$ & $\mathrm{K}$ & $\mathrm{Cl}$ & $\mathrm{HCO}_{3}$ & $\mathrm{SO}_{4}$ \\
\hline$(\mathrm{cm})$ & \multicolumn{7}{|c|}{$\mathrm{cmol} \cdot \mathrm{L}^{-1}$} \\
\hline $0-10$ & $3,2 \mathrm{a}$ & $5,6 \mathrm{a}$ & 6,2 a & $3,1 \mathrm{a}$ & $6,2 \mathrm{a}$ & $2,6 a$ & $5,5 \mathrm{a}$ \\
\hline $10-20$ & $2,1 \mathrm{~b}$ & $3,5 \mathrm{~b}$ & 4,7 a & 4,9 a & 5.8 a & $2,5 \mathrm{a}$ & 5,3 a \\
\hline $20-30$ & $2,3 \mathrm{~b}$ & $3,5 \mathrm{~b}$ & 5,8 a & $2,2 \mathrm{a}$ & 6.5 a & $1,9 \mathrm{a}$ & 3,3 a \\
\hline $30-40$ & $2,3 \mathrm{~b}$ & $3,3 \mathrm{~b}$ & 5,2 a & $2,6 \mathrm{a}$ & $6.7 \mathrm{a}$ & $1,7 \mathrm{a}$ & $3,4 \mathrm{a}$ \\
\hline $40-50$ & $2,0 \mathrm{~b}$ & $3,4 \mathrm{~b}$ & 7,4 a & 2,9 a & $7.0 \mathrm{a}$ & $1,5 \mathrm{a}$ & $2,8 \mathrm{a}$ \\
\hline \multirow[t]{2}{*}{ promedio } & 2,38 & 3,86 & 5,86 & 3,14 & 6,44 & 2,04 & 4,06 \\
\hline & \multicolumn{7}{|c|}{ Localidad 2: Puntos de muestreo 14 al 21} \\
\hline $0-10$ & $11,9 \mathrm{a}$ & $15,9 \mathrm{a}$ & $44,4 \mathrm{a}$ & $17,5 \mathrm{a}$ & $69,7 \mathrm{a}$ & $2,8 \mathrm{a}$ & 9,7 a \\
\hline $10-20$ & 9,1 a & $17,4 \mathrm{a}$ & 30,9 a & 23,7 a & $54,4 \mathrm{ab}$ & $1,9 \mathrm{ab}$ & 9,3 a \\
\hline $20-30$ & $12,1 \mathrm{a}$ & $22,7 \mathrm{a}$ & 39,6 a & $19,3 \mathrm{a}$ & $59,9 \mathrm{ab}$ & $2,0 \mathrm{ab}$ & 9,1 a \\
\hline $30-40$ & $8,0 \quad a$ & $14,9 \mathrm{a}$ & 37,6 a & $15,2 \mathrm{a}$ & $52,0 \mathrm{~b}$ & $1,5 \mathrm{ab}$ & 5,3 a \\
\hline $40-50$ & 9,4 a & $12,9 \mathrm{a}$ & $31,3 \mathrm{a}$ & $13,0 \mathrm{a}$ & $49,6 \mathrm{~b}$ & $1,2 \mathrm{~b}$ & $8,4 \mathrm{a}$ \\
\hline \multirow[t]{2}{*}{ promedio } & 10,08 & 16,76 & 36,7 & 17,74 & 57,12 & 1,88 & 8,36 \\
\hline & \multicolumn{7}{|c|}{ Localidad 3: Puntos de muestreo 22 al 29} \\
\hline $0-10$ & 20,1 a & $19,1 \mathrm{a}$ & 46,9 a & $42,0 \mathrm{a}$ & 86,6 a & $5,5 \mathrm{a}$ & $29,7 \mathrm{a}$ \\
\hline $10-20$ & $12,9 \mathrm{a}$ & $14,1 \mathrm{ab}$ & $38,8 \mathrm{~b}$ & 41,6 a & $63,4 \mathrm{ab}$ & $4,0 \mathrm{a}$ & $10,9 \mathrm{a}$ \\
\hline $20-30$ & $16,1 \mathrm{a}$ & $10,5 \mathrm{~b}$ & $36,7 \mathrm{~b}$ & 22,9 a & $50,8 \mathrm{~b}$ & $7,1 \mathrm{a}$ & $11,5 \mathrm{a}$ \\
\hline $30-40$ & $12,5 \mathrm{a}$ & $12,6 \mathrm{ab}$ & $34,1 \mathrm{~b}$ & $20,0 \mathrm{a}$ & $42,7 \mathrm{~b}$ & $7,2 \mathrm{a}$ & $12,1 \mathrm{a}$ \\
\hline \multirow[t]{2}{*}{ promedio } & 15,4 & 14,07 & 39,12 & 31,6 & 60,8 & 4,6 & 16,05 \\
\hline & \multicolumn{7}{|c|}{ Localidad 4: Puntos de muestreo 30 al 39} \\
\hline $0-10$ & $10,0 \mathrm{a}$ & $18,2 \mathrm{a}$ & $31,9 a$ & $32,8 \mathrm{a}$ & $64,3 \mathrm{a}$ & $4,6 \mathrm{a}$ & $7,6 \mathrm{a}$ \\
\hline $10-20$ & 7,7 a & $15,4 \mathrm{a}$ & $30,8 \mathrm{a}$ & $35,1 \mathrm{a}$ & $59,1 \mathrm{a}$ & $3,2 \mathrm{a}$ & $6,2 \mathrm{a}$ \\
\hline $20-30$ & 8,6 a & $10,4 \mathrm{a}$ & $35,9 a$ & $21,9 a$ & $57,2 \mathrm{a}$ & $5,0 \mathrm{a}$ & $6,2 \mathrm{a}$ \\
\hline $30-40$ & $7,3 \quad \mathrm{a}$ & $12,0 \mathrm{a}$ & $31,1 \mathrm{a}$ & $25,5 \mathrm{a}$ & $58,1 \mathrm{a}$ & $3,9 \mathrm{a}$ & $7,0 \mathrm{a}$ \\
\hline $40-50$ & 8,9 a & $13,4 \mathrm{a}$ & $40,7 \mathrm{a}$ & $33,0 \mathrm{a}$ & $72,2 \mathrm{a}$ & $3,8 \mathrm{a}$ & $8,5 \mathrm{a}$ \\
\hline promedio & 8,5 & 13,88 & 34,08 & 23,66 & 62,18 & 4,1 & 7,1 \\
\hline
\end{tabular}

Letras diferentes denotan diferencias significativa al $5 \%$ por la prueba de Tukey.

En estas condiciones, donde los mantos freáticos se muestran entre 0 a $0,5 \mathrm{~m}$ y 0,5 a $1 \mathrm{~m}$ y la conductividad eléctrica oscila entre 0,51 y $53,2 \mathrm{dS} \mathrm{m}^{-1}$, por procesos de ascenso capilar, los iones $\mathrm{Na}, \mathrm{Cl}, \mathrm{SO}_{4}$ y $\mathrm{Mg}$ asciendan a los horizontes superficiales, favorecidos por la alta evapotranspiración de la zona, fenómenos que contribuyen con la formación y la precipitación química de diferentes tipos de sales. Qadir et al. (2007) exponen que las sales solubles predominantes en los suelos salinos son cloruros, sulfatos y bicarbonatos de $\mathrm{Na}$, Ca y Mg.

Para la localidad (L3), en el periodo lluvioso, los niveles $\mathrm{Cl}$ y $\mathrm{Mg}$ descendieron a niveles medios; $\mathrm{Ca}$ y $\mathrm{HCO}_{3}$, a niveles bajos, mientras que los niveles de $\mathrm{SO}_{4}$, pasaron de medios, en el periodo seco, a altos, en el periodo lluvioso. Al igual que 
en el periodo seco, los máximos valores de estos iones, se presentan en la profundidad de $0-10 \mathrm{~cm}$ (Tabla 3).
En términos generales, la localidad L1, se caracterizó por mostrar las concentraciones más bajas de iones y L3, por los

Tabla 3. Concentración de iones formadores de sales para diferentes localidades y profundidades de suelos, de la desembocadura del río Sinú, en periodo lluvioso, entre 2002 y 2007.

\begin{tabular}{|c|c|c|c|c|c|c|c|}
\hline \multicolumn{8}{|c|}{ Localidad 1: Puntos de muestreo 1 al 13} \\
\hline profundidad & $\mathrm{Ca}$ & $\mathrm{Mg}$ & $\mathrm{Na}$ & $\mathrm{K}$ & $\mathrm{Cl}$ & $\mathrm{HCO}_{3}$ & $\mathrm{SO}_{4}$ \\
\hline$(\mathrm{cm})$ & \multicolumn{7}{|c|}{$\mathrm{cmol} \cdot \mathrm{L}^{-1}$} \\
\hline $0-10$ & $1,5 \mathrm{a}$ & $3,5 \mathrm{a}$ & $3,3 \mathrm{a}$ & $2,7 \mathrm{a}$ & $3,2 \mathrm{~b}$ & 3,1 a & 4,8 a \\
\hline $10-20$ & $1,5 \mathrm{a}$ & 3,3 a & $2,5 \mathrm{a}$ & $1,7 \mathrm{a}$ & $3,0 \mathrm{~b}$ & $2,4 \mathrm{ab}$ & $3,9 \mathrm{a}$ \\
\hline $20-30$ & $1,7 \mathrm{a}$ & $3,7 \mathrm{a}$ & $2,8 \mathrm{a}$ & $1,8 \mathrm{a}$ & $3,4 \mathrm{~b}$ & $2,4 \mathrm{ab}$ & $4,3 \mathrm{a}$ \\
\hline $30-40$ & $1,8 \mathrm{a}$ & $6,8 \mathrm{a}$ & $3,2 \mathrm{a}$ & $1,9 \mathrm{a}$ & $4,4 \mathrm{a}$ & $2,5 \mathrm{ab}$ & $4,4 \mathrm{a}$ \\
\hline $40-50$ & $1,8 \mathrm{a}$ & $3,4 \mathrm{a}$ & $3,4 \mathrm{a}$ & $1,2 \mathrm{a}$ & $5,3 \mathrm{a}$ & $1,6 \mathrm{~b}$ & $3,3 \mathrm{a}$ \\
\hline \multirow[t]{2}{*}{ promedio } & 1,66 & 4,14 & 3,04 & 1,86 & 3,86 & 2,40 & 4,14 \\
\hline & \multicolumn{7}{|c|}{ Localidad 2: Puntos de muestreo 14 al 21} \\
\hline $0-10$ & $10,1 \mathrm{a}$ & 15,6 a & $22,0 \mathrm{a}$ & $10,1 \mathrm{a}$ & $44,7 \mathrm{a}$ & $3,2 \mathrm{a}$ & $14,7 \mathrm{a}$ \\
\hline $10-20$ & $7,3 \mathrm{ab}$ & $14,0 \mathrm{a}$ & $21,6 \mathrm{a}$ & $6,4 \mathrm{a}$ & $38,1 \mathrm{a}$ & $3,0 \mathrm{a}$ & $14,7 \mathrm{a}$ \\
\hline $20-30$ & 6,9 a & 13,4 a & 19,2 a & 6,0 a & 36,5 a & $2,9 \mathrm{a}$ & $15,1 \mathrm{a}$ \\
\hline $30-40$ & $7,2 \mathrm{ab}$ & $13,7 \mathrm{a}$ & $20,3 \mathrm{a}$ & $8,4 \mathrm{a}$ & 35,5 a & $3,0 \mathrm{a}$ & $14,0 \mathrm{a}$ \\
\hline $40-50$ & $9,0 \mathrm{ab}$ & $14,3 \mathrm{a}$ & $20,5 \mathrm{a}$ & $9,7 \mathrm{a}$ & $41,7 \mathrm{a}$ & $2,4 \mathrm{a}$ & 9,3 a \\
\hline \multirow[t]{2}{*}{ promedio } & 8,1 & 14.2 & 20,7 & 8,1 & 39,3 & 2,9 & 13,56 \\
\hline & \multicolumn{7}{|c|}{ Localidad 3: Puntos de muestreo 22 al 29} \\
\hline $0-10$ & $6,50 \mathrm{a}$ & 16,03 a & 50,39 a & 11,96 a & 63,6 a & 3,65 a & $34,11 \mathrm{a}$ \\
\hline $10-20$ & 5,65 a & 12,86 a & 40,36 a & 8,89 a & $44,7 \mathrm{ab}$ & $2,92 \mathrm{ab}$ & 31,13 a \\
\hline $20-30$ & 7,73 a & $13,28 \mathrm{a}$ & $32,84 \mathrm{a}$ & 9,64 a & $47,9 a b$ & $2,31 \mathrm{~b}$ & $17,26 \mathrm{a}$ \\
\hline $30-40$ & 5,78 a & $14,92 \mathrm{a}$ & 29,27 a & $10,24 \mathrm{a}$ & $46,3 \mathrm{ab}$ & $2,52 \mathrm{~b}$ & $12,97 \mathrm{a}$ \\
\hline $40-50$ & $5,90 \mathrm{a}$ & $13,44 \mathrm{a}$ & 29,59 a & $11,71 \mathrm{a}$ & 39,3 a & $2,35 \mathrm{~b}$ & $19,38 \mathrm{a}$ \\
\hline \multirow[t]{2}{*}{ promedio } & 6,31 & 14,10 & 36,49 & 10,48 & 48,3 & 2,75 & 22,97 \\
\hline & \multicolumn{7}{|c|}{ Localidad 4: Puntos de muestreo 30 al 39} \\
\hline $0-10$ & $4,88 \mathrm{a}$ & 8,66 a & $19,74 \mathrm{a}$ & 7,90 a & 34,00 a & 3,32 a & 8,76 a \\
\hline $10-20$ & $4,97 \mathrm{a}$ & $8,10 \mathrm{a}$ & 17,53 a & 7,58 a & 33,33 a & $2,91 \mathrm{ab}$ & $6,54 \mathrm{a}$ \\
\hline $20-30$ & $5,87 \mathrm{a}$ & 9,24 a & 18,19 a & $9,52 \mathrm{a}$ & 36,45 a & $2,86 a b$ & 8,23 a \\
\hline $30-40$ & 4,93 a & $7,01 \mathrm{a}$ & $15,50 \mathrm{a}$ & 5,09 a & 36,41 a & $2,43 \mathrm{ab}$ & $8,87 \mathrm{a}$ \\
\hline $40-50$ & 6,85 a & $10,87 \mathrm{a}$ & $20,37 \mathrm{a}$ & 9,63 a & $45,77 \mathrm{ab}$ & $2,22 \mathrm{~b}$ & $12,34 \mathrm{ab}$ \\
\hline promedio & 5,50 & 8,77 & 18,45 & 7,94 & 37,19 & 2,74 & 8,94 \\
\hline
\end{tabular}

Letras diferentes denotan diferencias significativa al $5 \%$ por la prueba de Tukey. 
contenidos máximos de iones formadores de sales, en los periodos seco y lluvioso, mientras que las localidades L2 y L4, presentan valores de transición.

De igual forma, con excepción del $\mathrm{SO}_{4}$, los contenidos de los iones $\mathrm{Na}$ y $\mathrm{Cl}$ decrecen en el periodo lluvioso, condición que contribuye a la disminución de la salinidad de estos suelos
(Tabla 4). La disminución de $\mathrm{Na} \mathrm{y} \mathrm{Cl}$, se puede explicar por la disolución de estos iones con el incremento en los volúmenes de agua, en esta época, ya que la hidroeléctrica de Urrá I, entre febrero y marzo, aporta volúmenes de agua de 170,2 y $199 \mathrm{~m}^{3} \mathrm{~s}^{-1}$ y, en los meses de alta precipitación, octubre y noviembre, 464,9 y $361,3 \mathrm{~m}^{3} \mathrm{~s}^{-1}$.

Tabla 4. Media general de iones estudiados en el extracto de saturación en las diferentes localidades del área de influencia, de la desembocadura del río Sinú, entre 2002 y 2007.

\begin{tabular}{|c|c|c|c|c|c|}
\hline \multicolumn{7}{|c|}{ Periodo Seco: Diciembre - Abril } \\
\hline & L1 & L2 & L3 & L4 & Contenidos de iones \\
\hline $\mathbf{N a}$ & 7,18 & 34,81 & 45,35 & 34,13 & L3 > L2 > L4 > L1 \\
\hline $\mathbf{C l}$ & 7,16 & 59,36 & 64,26 & 62,21 & L3 > L4 > L2 > L1 \\
\hline $\mathbf{M g}$ & 3,92 & 18,83 & 15,50 & 13,92 & L2 > L3 > L4 > L1 \\
\hline $\mathbf{C a}$ & 2,42 & 10,54 & 12,49 & 8,54 & L3 > L2 > L4 > L1 \\
\hline $\mathbf{S O}_{4}$ & 4,10 & 8,41 & 14,57 & 7,16 & L3 > L2 > L4 > L1 \\
\hline $\mathrm{HCO}_{3}$ & 2,08 & 1,94 & 7,27 & 4,14 & L3 > L4 > L1 > L2 \\
\hline \multicolumn{7}{|c|}{ Periodo Lluvioso: Mayo - Noviembre } \\
\hline $\mathrm{Na}$ & 3,08 & 20,73 & 36,49 & 18,67 & L3 > L2 > L4 > L1 \\
\hline $\mathrm{Cl}$ & 3,90 & 39,37 & 48,37 & 37,19 & L3 > L2 > L4 > L1 \\
\hline $\mathbf{M g}$ & 4,17 & 14,24 & 14,41 & 8,77 & L3 > L2 > L4 > L1 \\
\hline $\mathrm{Ca}$ & 1,72 & 8,16 & 6,31 & 5,50 & L2 > L3 > L4 > L1 \\
\hline $\mathrm{SO}_{4}$ & 4,19 & 13,59 & 22,97 & 8,95 & L3 > L2 > L4 > L1 \\
\hline $\mathrm{HCO}_{3}$ & 2,42 & 2,92 & 2,75 & 2,75 & L2 > L3 = L4 > L1 \\
\hline
\end{tabular}

En la tabla 5, se indica la correlación de Pearson, entre los valores de conductividad eléctrica y las concentraciones de iones, determinados a cada profundidad, en periodos lluviosos y secos. Esta correlación, para las diferentes profundidades, en el periodo seco, indica que, con excepción del $\mathrm{HCO}_{3}$, los iones $\mathrm{Cl}$, $\mathrm{Na}$ y $\mathrm{Mg}$ registraron correlaciones positivas y altamente significativas con la conductividad eléctrica, con $r \geq 0,77^{* *}, r \geq 0,75^{* *}$ y $r \geq 0,73^{* *}$; sin embargo, la concentración de Na y Mg disminuyó en el periodo lluvioso y aumentó la concentración de los iones $\mathrm{Cl}$, Ca y $\mathrm{SO}_{4}$.

En estas condiciones es importante anotar que la salinidad en el periodo seco es de mayor representatividad, debido a que se presentan conductividades eléctricas mayores de 2 $\mathrm{dS} \mathrm{m}^{-1}$, ya que en este período, las sales están más concentradas y manifiestan todo su potencial, mientras que en el periodo lluvioso, se encuentran diluidas (Porta et al.1999). Kurtulus et al. (2009) indican que la conductividad eléctrica del suelo es función del contenido de arcilla, contenido de agua y la salinidad. Según Ghassemi et al. (1995), las principales causas potenciales de salinización cerca de la superficie del suelo, se pueden conseguir por la migración de agua salina, a través de la intrusión de agua de mar en las zonas costeras y por la concentración y evaporación de las aguas subterráneas poco profundas, que han surgido en respuesta al riego o ascenso de aguas freáticas.

De los resultados obtenidos, se puede concluir que el área de suelos no salinos y ligeramente salinos, se establecieron en el orden del 51,2 al 80,8\% en los periodos estudiados, en la localidad 1(L1), que es la zona de mar altimetría y la más alegada del océano Atlántico; además, a medida que se profundiza en el suelo, se localizan texturas francas a francas arenosas, que contribuyen con el lavado de sales.

Por otra parte y de acuerdo con la distribución espacial, se apreció que el área de suelos con salinidad media a extremadamente salinos, se presentan en la localidad 3 (L3), con 
Tabla 5. Correlación simple (Pearson), entre CE e iones estudiados en el extracto de saturación para diferentes profundidades del área de influencia, de la desembocadura del río Sinú, entre 2002 y 2007.

\begin{tabular}{|c|c|c|c|c|c|c|c|c|}
\hline \multicolumn{9}{|c|}{ Periodo Seco: Diciembre - Abril } \\
\hline & & $\mathrm{Ca}$ & $\mathrm{Mg}$ & $\mathrm{Na}$ & $\mathrm{K}$ & $\mathrm{Cl}$ & $\mathrm{HCO}_{3}$ & $\mathrm{SO}_{4}$ \\
\hline $0-10$ & \multirow{5}{*}{ CE } & $0,54 * *$ & $0,81 * *$ & $0,78 * *$ & $0,31 *$ & $0,77 * *$ & $0,11 \mathrm{~ns}$ & $0.22 * *$ \\
\hline $10-20$ & & $0,44 * *$ & $0,79 * *$ & $0,83 * *$ & $0,69 * *$ & $0,93 * *$ & $0,10 \mathrm{~ns}$ & $0.16 *$ \\
\hline $20-30$ & & $0,71 * *$ & $0,92 * *$ & $0,75^{* *}$ & $0,46 * *$ & $0,76 * *$ & $0,05 \mathrm{~ns}$ & $0.21 *$ \\
\hline $30-40$ & & $0,30 * *$ & $0,73 * *$ & $0,85^{* *}$ & $0,50 * *$ & $0,91 * *$ & $0,04 \mathrm{~ns}$ & $0.14 \mathrm{~ns}$ \\
\hline $40-50$ & & $0,43 * *$ & $0,84 * *$ & $0,86 * *$ & $0,39 * *$ & $0,92 * *$ & $0,07 \mathrm{~ns}$ & $0.33 * *$ \\
\hline \multicolumn{9}{|c|}{ Periodo Lluvioso: Mayo - Noviembre } \\
\hline $0-10$ & \multirow{5}{*}{ CE } & $0,71 * *$ & $0,84 * *$ & $0,74 * *$ & $0,41 * *$ & $0,91 * *$ & 0,13 ns & $0.55 * *$ \\
\hline $10-20$ & & $0,76 * *$ & $0,84 * *$ & $0,73 * *$ & $0,42 * *$ & $0,95 * *$ & $0,16 \mathrm{~ns}$ & $0.52 * *$ \\
\hline $20-30$ & & $0,77 * *$ & $0,79 * *$ & $0,75^{* *}$ & $0,55^{* *}$ & $0,94 * *$ & $0,19 *$ & $0.65 * *$ \\
\hline $30-40$ & & $0,58 * *$ & $0,53 * *$ & $0,62 * *$ & $0,41^{* *}$ & $0,95 * *$ & $0,16 \mathrm{~ns}$ & $0.48 * *$ \\
\hline $40-50$ & & $0,69 * *$ & $0,78 * *$ & $0,70 * *$ & $0,56 * *$ & $0,91 * *$ & $0,19 *$ & $0.61 * *$ \\
\hline
\end{tabular}

*Significativo al $5 \%$, ** significativo al $1 \%$ y ns no significativo

un 19,2 y $48,8 \%$ y los iones que dominan en estas condiciones, son $\mathrm{Na}, \mathrm{Cl}, \mathrm{y} \mathrm{Mg}$, principalmente, en el periodo seco. Esta concentración de sales es explicada, debido a que es el área más próxima a la desembocadura del río Sinú, donde se despliegan zonas de baja altimetría, acompañadas de texturas francas, con mayor contenido de arcillas. Se recomienda replicar en un ciclo bianual, los monitoreos de salinidad en aguas freáticas y de suelos, después de haber suspendido los estudios.

Agradecimientos: Al proyecto de investigación "Diagnóstico general del nivel de salinización en la zona aledaña a la desembocadura del río Sinú y su impacto socioeconómico: Período Abril/2002 - noviembre/2008" y al personal técnico del Laboratorio de Suelos y Aguas de la Facultad de Ciencias Agrícolas de la Universidad de Córdoba, Colombia. Conflicto de intereses: Los autores declaramos que no existe ningún conflicto de interés, que ponga en riesgo la validez de los resultados presentados.

\section{BIBLIOGRAFÍA}

1. AMEZKETA, E. 2006. Problemática relacionada con la salinidad del suelo en Navarra. Disponible desde Internet en: http://www.navarra.es/NR/ rdonlyres/DC788C10-FD10-4CAB-9829 67EAC828A8C6/0/NavarraSalinidadSuelos.pdf (con acceso $04 / 07 / 2011)$.
2. ALLAIRE, S.E.; LANGE, S.F.; LAFOND, J.A.; PELLETIER, B.; CAMBOURIS, A.N.; DUTILLEUL, P. 2012. Multiscale spatial variability of $\mathrm{CO}_{2}$ emissions and correlations with physico-chemical soil properties. Geoderma (Netherlands). 170:251-260.

3. BAYUELO-JIMÉNEZ, J.S.; CRAIG, R.; LYNCH, J.P. 2002. Salinity tolerance of Phaseolus species during germination and early seedling growth. Crop Sci. 42:15841594.

4. CIAF. 1985. Estudios básicos para un plan de ocupación del espacio de la Cuenca del Río Sinú. Departamento Nacional de Planeación. Corporación Autónoma Regional de los valles del Sinú y San Jorge, CVS. Bogotá, Colombia. 328p.

5. $\mathrm{CIOH}$. 1994. Centro de Investigaciones Oceanográficas e Hidrológicas. Estudio oceanográfico de recuperación de playas sector La Perdiz Puerto Viejo. Golfo de Morrosquillo. Cartagena de Indias D.T. y C. 189p.

6. FAO. 2005. Management of irrigation-induced salt-affected soils. Joint publication of CISEAU, IPTRID and FAO, Rome. p.34-65. 
7. FAO, 2008. Land and Plant Nutrition Management Service. Disponible desde Internet en: http://www.fao.org/ ag/agl/agll/spush (con acceso 24/05/2012).

8. GHASSEMI, F.; JAKEMAN, A.J.; NIX, H.A. 1995. Salinization of Land and Water Resources, CAB Int., Wallingford, U.K. 526p.

9. GIRÓN-RÍOS, Y.; OLESCHKO-LUTKOVA, K.; JEANFRANCOIS, P.; HERNÁNDEZ-ALCÁNTARA, M.; CAMARILLO GARCÍA, E.; VELÁZQUEZ-GARCÍA, J. 2009. Análisis fractal de la reflectancia de los suelos salinos. Agrociencia. 43:403-416.

10. GONÇALVES, I.V.C.; FREIRE, M.B.G. dos S.; SANTOS, M.A.; SOUZA, E.R.; FREIRE, F.J. 2011. Alterações químicas de um Neossolo Flúvico irrigado com águas salinas. Rev. Ciência Agronôm. 42(3):589596.

11. HOLDRIDGE, R. 1978. Ecología basada en zonas de vida. IICA Libros y Materiales Educativos, 34p.

12. IDEAM. 2002. Zonificación de los procesos de salinización de los suelos de Colombia. Instituto de Hidrología, Meteorología y Estudios Ambientales - IDEAM, Subdirección de Geomorfología y Suelos. 44p.

13. JENKS, M.A.; HASEGAWA, P.M. 2005. Plant Abiotic Stress. India. Blackwell Publishing Ltd. 270p.

14. JOHANN, J.A.; OPAZO, M.A.U.; SOUZA, E.G.; ROCHA, J.V. 2004. Variabilidade espacial dos atributos físicos do solo e da produtividade em um Latossolo Bruno distrófico da região de Cascavel, PR. Rev. Bras. Engenh. Agri. Ambiental. 8(2-3):212-219.

15. JUAN, P.; MATEU, J.; JORDAN, M.M.; MATAIX-SOLERA, J.; MELÉNDEZ-PASTOR, I.; NAVARRO-PEDREÑO, J. 2011. Geostatistical methods to identify and map spatial variations of soil salinity. J. Geochem. Explor. (USA). 108(1):62-72.

16. KURTULUS, C.; CANBAY, M.; DEMIR, N.; GIDER, D. 2009. Salinity investigation of the region east to the Izmit Gulf in Izmit-Kocaeli, J. Food Agric. Environ. 7(2):755-758.

17. OTERO, J.; GÓMEZ, C.; SÁNCHEZ, R. 2002. Zonificación de los procesos de salinizacion de los suelos de Colombia. Subdirección de Geomorfología y Suelos. IDEAM. 44p.
18. PALENCIA, G.; MERCADO, T.; COMBATT, E. 2006. Estudio agroclimático del departamento de Córdoba. Facultad de Ciencias Agrícolas, Universidad de Córdoba. 126p.

19. PERFETTI, P.B.; TERREL, C.R. 1989. Water quality indicators guide: Surface waters. USDA Misc. Publ. SCSTP-161. Washington, DC: U.S. Government Print Office. 98p.

20 .PORTA, J. 2007. Salinización y sodificación: suelos de regadío. En: Porta, J. (ed). Edafología para la agricultura y el medio ambiente, Mundi-Prensa, Madrid. $657 p$.

21. PORTA, J.; LÓPEZ-ACEVEDO, M.; ROQUERO, C. 1999. Edafología para la Agricultura y el Medio Ambiente. $2^{\mathrm{a}}$ ed. Mundi Prensa. Madrid. 420p.

22. QADIR, M.; OSTER, J.D.; SCHUBERT, S.; NOBLE, A.D.; SAHRAWAT, K.L. 2007. Phytoremediation of sodic and saline-sodic soils. Adv. Agronomy. 96:197-247.

23. QUEIROZ, J.E.; GONÇALVES, A.C.A.; SOUTO, J.S.; FOLEGATTI, M.V. 2010. Avaliação e monitoramento da salinidade do solo. In: Gheyi, H.R.; Dias, N. da S.; Lacerda, C.F. (eds) Manejo da salinidade na agricultura. Fortaleza: INCT Sal. 472p.

24. RAMOLIYA, P.J.; PATEL, H.M.; PANDEY, A.N. 2006. Effect of salinization of soil on growth and nutrient accumulation in seedlings of Prosopis cineraria. J. Plant Nutr. 29:283-303.

25. RANGEL-CH, J.O.; ARELLANO, H. 2010. Clima. En: Rangel-C., J.O. (ed). Diversidad Biótica IX. Ciénagas de Córdoba: Biodiversidad, Ecología y Manejo ambiental. Instituto de Ciencias Naturales. Universidad Nacional de Colombia. p 1-14.

26. RENGASAMY, P. 2006. World salinization with emphasis on Australia. J. Exp. Bot. 57(5)1017-1023.

27. RICHARDS, L. 1954. Diagnosis and improvement of saline and alkali soils. Washintong: USSL. USDA Handbook 60. 160p.

28. RIDD, P.V.; STIEGLITZ, T. 2002. Dry season salinity changes in arid estuaries fringed by mangroves and salt flats. Estuarine Coastal Shelf Sci. 54:1039-1049. 
29. RYCROFT, D.W.; KYEI-BaFFOUR, N.; TANTON, T. 2002. Recibido: Febrero 14 de 2014

The effect of sodicity on the strength of a soil surfa- Aceptado: Agosto 15 de 2014 ce. Irrigation and Drainage. 51:339-346.

30. SÁNCHEZ, H.; ÁLVAREZ, R.; PINTO, F.; SÁNCHEZ, A.; PINO, J.; GARCÍA, I.; ACOSTA, M. 1997. Diagnóstico y zonificación preliminar de los manglares del Caribe de Colombia. Proy. PD 171/91 Rev. 2 (F) Fase I. Conservación y manejo para el uso múltiple y el desarrollo de los manglares de Colombia, MADT/ OIMT. Santa Fe de Bogotá D.C., Colombia. 511p.

\section{Como citar:}

Narváez M., H.; Combatt C., E.; Bustamante Barrera, I. 2014. Distribución espacial de la salinidad en suelos del área de influencia de la desembocadura del rio Sinú (Córdoba, Colombia). Rev. U.D.C.A Act. \& Div. Cient. 17(2): 433-443. 